Freiwillige Umwelt- und Sozialstandards auf Erfolgskurs

\section{Verwirrende Regulierung oder echte Alternativen}

\author{
Produkte aus fairem Handel und dem Ökolandbau haben in \\ den letzten Jahren den Durchbruch auf den Märkten geschafft. \\ Die Herstellung dieser Produkte basiert oft auf freiwilligen Ver- \\ einbarungen. So werden gegenwärtig Standards für Biokraft- \\ stoffe und Soja verhandelt. Doch was verbirgt sich hinter diesen \\ Umwelt- und Sozialstandards? Von Ines Freier
}

D ie wohl bekanntesten Standards im Bereich des privaten Konsums sind die Standards für den Ökolandbau oder die Standards für fairen Handel. Weiterhin gehören beispielsweise das Forest Steward Ship-Siegel (FSC) oder der Sozialstandard SA 8000 dazu. Eher unbekannte Standards sind der Marine Stewardship Council (MSC) oder das Flower Label Program (FLP).

In Deutschland findet man zunehmend Label, die aus dem angelsächsischen Raum kommen wie beispielsweise das Rainforest Alliance Label oder Utz Kapeh für die Kaffeeproduktion. Diese Standards haben einen privatwirtschaftlichen Charakter, das heißt Unternehmen setzen sich selbst Verhaltensnormen. Diese Standards sind also, ähnlich den Vorgaben der Internationalen Organisation für Normung (ISO), freiwillig. Sie nehmen jedoch oft Bezug auf staatliche Normen wie die nationale Gesetzgebung im Bereich Umwelt oder Arbeit und internationale Abkommen wie die Kernarbeitsnormen der Internationalen Arbeitsorganisation (ILO), die als Mindestanforderungen gelten. Die Besonderheit der freiwilligen Standards gegenüber den ISO-Normen ist, dass sie in vielen Fällen als Multistakeholder-Initiativen umgesetzt werden.

\section{Vielfalt der Standards}

Einige dieser freiwilligen Standardinitiativen haben sich zusammengeschlossen und gründeten als Dachorganisation
The International Social and Environmental Accreditation and Labelling Alliance (ISEAL) mit Sitz in London (1). Diese vergibt kein eigenes Label, sondern unterstützt die einzelnen freiwilligen Standards. Weltweit haben mindestens 20.000 Unternehmen oder Genossenschaften die Standards unter dem Dach von ISEAL umgesetzt. Die Ökolandbaubetriebe können dabei aus statistischen Gründen nicht mitgezählt werden, denn es gibt kein zentrales Register für zertifizierte Unternehmen. Im Vergleich dazu verzeichnete die ISO $14001 \mathrm{im} \mathrm{Jahr} 2006$ ungefähr 100.000 zertifizierte Organisationen.

Die freiwilligen Standards lassen sich grob anhand von zwei Kategorien, Zielmärkte und Zielkriterien, klassifizieren. Die Fair Trade Standards haben lange auf alternative Märkte in Industrieländern gezielt. Alle anderen Standards haben von Anfang an auf den Massenmarkt gesetzt. Hinsichtlich der Zielkriterien kann man zwischen überwiegend ökologischen Zielen wie Schutz der Artenvielfalt und überwiegend sozialen Zielen wie die Einhaltung der ILO-Arbeitsnormen unterscheiden.

\section{Unterschiedliche Anforderungen}

Die Anforderungen, die an Unternehmen gestellt werden, um das Siegel zu erhalten, sind unterschiedlich hoch und je nach Initiative verschieden. Die Unterschiede zwischen den beiden Kategorien sind auch nicht mehr scharf: Zum einen entdecken die Supermärkte den fairen Handel. Zum anderen fordern viele freiwillige Standards sowohl die Erfüllung von Umweltkriterien als auch von sozialen Kriterien. Weiterhin geht der Trend weg von Standards, die sich nur auf ein Produkt oder ein Kriterium beziehen, wie das bekannte Rugmark-Siegel für Teppiche ohne Kinderarbeit, hin zu breiteren Standards wie beispielsweise Utz Kapeh für die Kaffeeproduktion.

Die Initiativen sind auch unterschiedlich auf den Märkten sichtbar. Während viele Standards wie die Rainforest Alliance oder das Siegel für Kaffee Utz Kapeh Produktlabel sind, ist die Fair Labour Association, die die Einhaltung von Sozialstandards in der Textilproduktion fördert, bewusst nicht auf Märkten als Label präsent.

\section{Erfolgsbedingungen}

Seit Mitte der 1990er Jahre haben sich die meisten der neueren Initiativen herausgebildet, während es Ökolandbau und fairen Handel schon länger gibt. Erst in den letzten Jahren konnten sich jedoch die freiwilligen Standards auch auf dem Markt durchsetzen. Zwei wichtige Erfolgsbedingungen sind, dass sie erstens auf Trends in Massenmärkten eingehen und zweitens Multistakeholder-Initiativen sind.

Die Entwicklung ist beeindruckend, denn die Umsätze von Produkten aus fairem Handel stiegen in den letzten Jahren um durchschnittlich 40 Prozent, in Deutschland allein um 50 Prozent im Jahr 2006. Die Fair Trade Labelling Organisation, der Dachverband der Verbände des fairen Handels, schätzt, dass inzwischen 1,4 Millionen Produzenten vom fairen Handel profitieren. Auch der Umsatz von Bioprodukten stieg in Deutschland im Jahr 2006 um 40 Prozent.

Der Trend hin zu fairen oder nachhaltigen Produkten auf den Märkten in Industrieländern ergibt sich aus einer Winwin-Situation für Konsumenten und Handel. Das Bewusstsein der Konsumenten für fair gehandelte und gesunde Produkte ist gestiegen, nicht zuletzt durch das Engagement von Nichtregierungsor- 
ganisationen. Der Handel greift diesen Trend gern auf, denn die Zahlungsbereitschaft der Kunden für diese Produkte ist vorhanden. Das inzwischen breite Sortiment an Produkten hebt sich von den üblichen Markenprodukten ab.

\section{Faire Produkte im Supermarkt}

Die Supermarktketten sind die Motoren und Gewinner dieser Entwicklung. Ihre Umsätze an fairen Produkten steigen stark an und öffnen die Massenmärkte für Produkte, die unter Einhaltung von Umwelt- und Sozialstandards hergestellt worden sind. Die bisherigen Märkte wie Bioläden oder Eine-Welt-Läden, die bewusst als Alternative geschaffen wurden, haben ihre Grenzen erreicht. Ein weiterer Erfolgsfaktor ist auch der Einstieg von Großunternehmen, die in ihren Zuliefererketten Sozial- und Umweltstandards umsetzen und sich dies zertifizieren lassen.

Diese Standards sind meist Multistakeholder-Intitiativen, an denen sich Nichtregierungsorganisationen, Unternehmen und auch Vertreter der Politik aus Entwicklungs- und Industrieländern beteiligen. Sie widmen sich zunehmend auch weniger für Konsumenten sichtbaren Themen wie der nachhaltigen Sojaproduktion oder Biokraftstoffen.

\section{Offene Fragen}

Diese Trends bei den freiwilligen Standards werfen zahlreiche Fragen auf. Zunächst ist, wie bei allen Standards, unklar, wie sich in den Unternehmen die Performance hinsichtlich sozialer und Umweltkriterien durch die Umsetzung der Standards ändert. Wirkungsuntersuchungen, die über einzelne Fallstudien oder Label hinausgehen, fehlen bisher.

Die Landschaft der verschiedenen freiwilligen Labels und Standards ist in den letzten Jahren sehr unübersichtlich geworden. Die Vielfalt der Labels mit ihren unterschiedlichen Anforderungen ist für Konsumenten kaum mehr verständlich, sodass die Standards harmonisiert werden müssen. Die Glaubwürdigkeit der Stan- dards leidet auch durch die stetig zunehmende Anzahl an Labeln.

Der Übergang von Nischenmärkten zu den Massenmärkten bietet einerseits Entwicklungschancen, andererseits müssen diese Standards für alle Produzenten zugänglich sein und daher eher niedrige Anforderungen stellen. Ein entscheidender Punkt ist die Frage nach der Legitimität dieser freiwilligen Standards, denn sie haben einen ausschließlich privatwirtschaftlichen Charakter. Sie können also nicht als Ersatz für dringend notwendige internationale Abkommen zum Beispiel im Bereich Wald oder Sozialstandards dienen. Trotz der beeindruckenden Entwicklung der freiwilligen Standards bleiben also zahlreiche Fragen offen.

\section{Anmerkungen}

(1) Im Internet unter: http://www.isealalliance.org

\section{Literatur}

German Federal Ministry for Economic Co-operation and Development: Outcomes and Impacts of Standards and Standard Initiatives, Bonn 2007.

Müller, M. / Mouchnik, A./ Freier, I.: Freiwillige Standardisierungs- und Zertifizierungsansätze vor dem Hintergrund einer nachhaltigen Entwicklung. In: Baumast, A. / Pape, J.: Betriebliches Umweltmanagement. Stuttgart 2007.

Fairtrade Labelling Organizations International (Hrsg.): Shaping Global Partnerships. Annual Report. Bonn 2007.

AUTORIN + KONTAKT

Ines Freier ist Beraterin für freiwillige Standards und Ressourcenmanagement in Potsdam. E-Mail: kontakt@ines-freier.de

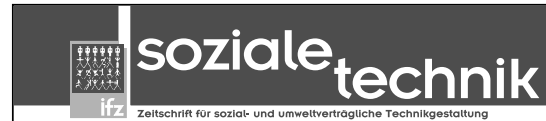

Seit 17 Jahren ist SOZIALE TECHNIK - herausgegeben vom Interuniversitären Forschungszentrum für Technik, Arbeit und Kultur (IFZ) - ein Forum für die interdisziplinäre Technikforschung und liefert Beiträge für eine sozial- und umweltverträgliche Gestaltung von Technologien. SOZIALE TECHNIK erscheint vierteljährlich, ein Jahresabonnement kostet $€ 18$,- (für Studierende $€ 13,-$ ).

Kostenloses Probeabonnement! Mit Beiträgen zu:

$\square$ Technologie \& Politik
$\square$ Umwelt \& Energie
$\square$ Neve Biotechnologien
$\square$ Frauen \& Technik
$\square$ Informations- \&

Kommunikationstechnologien soziale $_{\text {technik }}$

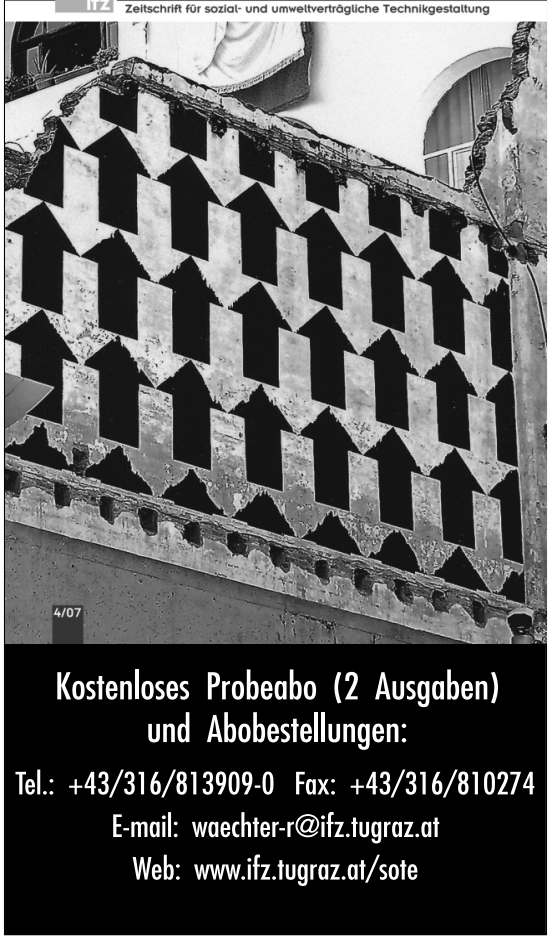


(c) 20I0 Authors; licensee IÖW and oekom verlag. This is an article distributed under the terms of the Creative Commons Attribution Non-Commercial No Derivates License (http://creativecommons.org/licenses/by-nc-nd/3.o/), which permits unrestricted use, distribution, and reproduction in any medium, provided the original work is properly cited. 